\title{
Nanotube Surface Arrays: Weaving, Bending, and Assembling on Patterned Silicon
}

\author{
Vladimir V. Tsukruk, ${ }^{*}$ Hyunhyub Ko, and Sergiy Peleshanko \\ Materials Science \& Engineering Department, Iowa State University, Ames, Iowa 50011, USA
}

(Received 6 August 2003; published 12 February 2004)

\begin{abstract}
We report the fabrication of ordered arrays of oriented and bent carbon nanotube on a patterned silicon surface with a micron scale spacing extending over millimeter size surface areas. We suggest that the patterning is controlled by the hydrodynamic behavior of a fluid front and orientation and bending mechanisms are facilitated by the pinned carbon nanotubes trapped by the liquid-solid-vapor contact line. The bending of the pinned nanotubes occurs along the shrinking receding front of the drying microdroplets. The formation of stratified microfluidic layers is vital for stimulating periodic instabilities of the contact line.
\end{abstract}

DOI: 10.1103/PhysRevLett.92.065502

Highly ordered arrays of carbon nanotubes assembled on solid surfaces are considered as a critical element for fabricating nanoscale electronic and electromechanical devices with a high density of organized functioning elements [1]. The unique shape of the carbon nanotubes and their bundles with the aspect ratio exceeding $1: 10^{4}$ are exploited for the fabrication of one-dimensional nanostructures such as randomly oriented monolayer films [2], oriented nematic arrays [3], arrays on patterned surface areas [4], precisely positioned individual nanotubes [5], and interelectrode functional networks [6]. The ordered growth of vertically oriented densely packed nanotubes on patterned surfaces has been observed $[7,8]$.

A high level of sophistication has been demonstrated with a variety of complex networks composed of straight carbon nanotubes. In-plane orientation was achieved by a variety of wet-chemistry approaches such as controlled directional fluidic flow, variable liquid-air contact line, selective adsorption on functionalized surfaces, as well as by the application of electric and magnetic fields [2$6,9,10]$. However, the fabrication of complex multidimensional networks requires the ability to control nanotube shapes considering a strong dependence of local electronic properties upon the bending state [11]. For example, the formation of the ring structure of biological macromolecules such as DNA is an efficient way to organize the bioinformation transfer [12].

It has been recently observed that nanotube rings can be formed by the nanotubes and their bundles during catalytic synthesis or oxidation process $[13,14]$. It has been suggested that the bending mechanism is based on thermal motion/bending or bubble cavitation. The resulting shape is stabilized by van der Waals interactions or chemical bonding between the tube ends. However, in all cases, only random collections of rings deposited on a solid substrate were observed.

Here we report a first successful result to produce a massive amount of straight and looped nanotubes (bent and folded nanotubes, open and closed loops) assembled with orientational order within positionally ordered arrays over millimeter size surface areas. This approach
PACS numbers: 61.46.+w, 81.07.-b

provides not only uniform orientation but also welldefined spacing in array positioning on a micron scale controlled by the dimensions of the alternating hydrophilic and hydrophobic surface areas. We suggest that the orientation and bending mechanism are facilitated by the pinned carbon nanotubes subjected to hydrodynamic patterned microflow followed by trapping at the liquid-solidvapor contact line undergoing the surface instability.

To facilitate the approach proposed, we used a combination of multistep purification and oxidation procedures to generate bundles containing 3-20 nanotubes of high purity. Selective adsorption was conducted on the surface of patterned silicon wafers. An oriented nanotube array was formed by casting or dip-coating techniques as shown in Fig. 1. The patterned surface was composed of alternating hydrophilic and hydrophobic stripes: amineterminated self-assembled monolayers (SAMs) with the ability for strong grafting of carbon nanotubes and methyl-terminated SAMs with weak adsorbing ability. Firm tethering can be caused by strong interaction between amine groups and sulfate and carboxylic groups of

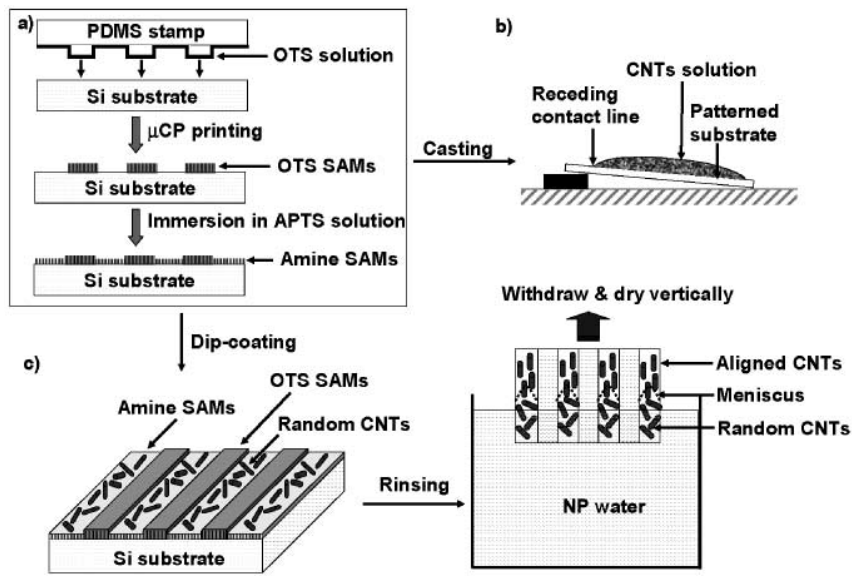

FIG. 1. (a) Schematic of the surface patterning through microcontact printing routine. (b) Casting of nanotube solution on tilted patterned substrate. (c) Dip coating. 
sodium dodecyl sulfate (SDS) molecules used to stabilize solution and nanotube ends $[15,16]$.

The variation of the withdrawing and casting conditions results in the fabrication of a variety of ordered arrays of carbon nanotubes anchored to hydrophilic, amine-terminated surface SAM areas represented by darker stripes of $7 \mu \mathrm{m}$ width (Fig. 2(d)). White stripes of about $3 \mu \mathrm{m}$ across on atomic force microscopy (AFM) topographical images represent thicker hydrophobic methyl-terminated areas (about $1.2 \mathrm{~nm}$ difference in thickness). The types of local orientation and ordering of the carbon nanotubes confined to the "sticky" stripes range from high density array of "woven" nanotube monolayer with different textures (chevron or more complicated), oriented straight nanotubes, bent nanotubes stretched along the stripes, and bent "combed" nanotubes (see some examples in Figs. 2 and 3). Orientation ordering within individual stripes resembles that achieved by unidirectional fluidic flow and can be described as nematic type with uniform local orientation and no longitudinal positional order as indicated by narrow arcs in a $2 \mathrm{D}$ Fourier transform image [Fig. 2(b)]. These oriented array areas with virtually an identical repetition of oriented texture within stripes are regularly spaced over millimeter size surface areas as confirmed by sharp, pointlike rows of corresponding Fourier transforms from fabricated arrays [Fig. 2(a)]. These nanotube arrays are firmly tethered to functionalized silicon surfaces through a combination of chemical bonding and strong van der Waals interactions [5,17] and cannot be removed with intensive ultrasonic treatment.

Careful analysis of the local configuration of carbon nanotubes within the surface arrays of low surface coverage unexpectedly discovered consistent repetition of similar, nonlinear shapes of individual nanotubes, which are very different from rodlike shapes of nanotubes aligned by uniform flow [Figs. 2(d), 2(e), and 3]. Depending upon specific fabrication conditions (rate of withdrawal, rinsing, and drying), sharply folded "needle eyes," elongated "hooks," single and double "nooses," and "arches" are consistently observed for nanotubes and their bundles on a micron scale (Fig. 3). The bent nanotubes are uniformly oriented along one direction although their positions within stripes are not correlated. On average, up to $90 \%$ of nanotubes are uniformly oriented and up to $40 \%$ of all aligned nanotubes under given conditions are folded in a similar manner with a high degree of shape persistence over large surface areas with linear density of about one nanotube per micron. This is a relatively high yield considering a stochastic nature of the process. This ordering could be further enhanced by, e.g., creating a rectangular grid of hydrophilic/hydrophobic surface areas. In fact, our preliminary results demonstrated that in this case, indeed, a regular array of "nest"-shaped carbon nanotubes predominantly aligned along the edges of hydrophilic squares can be created [Fig. 2(f)].

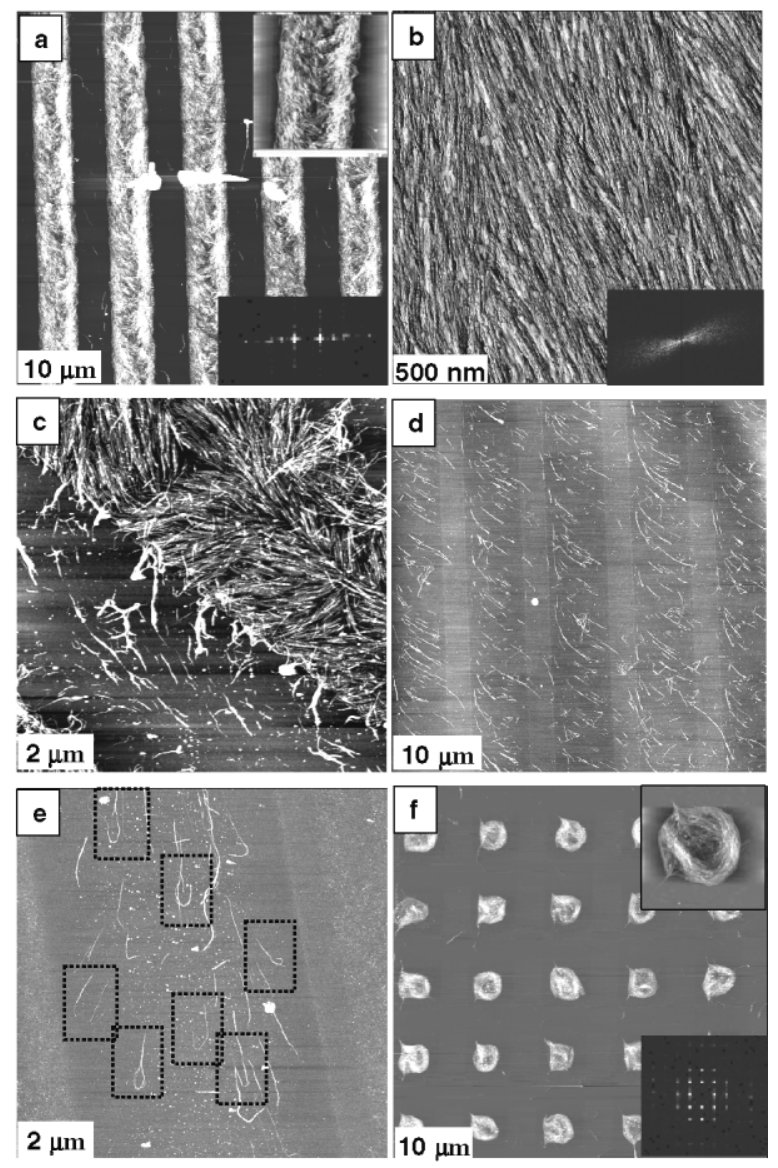

FIG. 2. AFM topographical [(a), (c)-(f)] and phase (b) images of different types of patterned surface arrays of woven and combed nanotube arrays formed by casting [(a), (b), (c), (f)] and by dip coating [(d), (e)]. (a) Woven nanotube arrays on parallel stripes, $50 \times 50 \mu \mathrm{m}$; top inset shows microstructure of a single stripe $(10 \times 10 \mu \mathrm{m})$; bottom inset shows 2D Fouriertransform demonstrating perfect spacing and vertical ordering; $Z$ scale is $40 \mathrm{~nm}$. (b) High resolution $(2 \times 2 \mu \mathrm{m})$ phase image of a highly oriented straight carbon nanotube array within a single stripe; inset shows 2D Fourier transform demonstrating a high level of orientation nematic ordering; $Z$ scale is $10^{\circ}$. (c) Image of an individual stripe $(10 \times 10 \mu \mathrm{m})$ with a complex chevron type of oriented nanotubes; $Z$ scale is $20 \mathrm{~nm}$. (d) Combed nanotube monolayer showing long-range orientation of bent tubes uniformly oriented within stripes; $Z$ scale is $20 \mathrm{~nm}$. (e) Selected surface areas with tethered carbon nanotubes in "noose" shapes. Marked areas show identically looped nanotubes; $Z$ scale is $20 \mathrm{~nm}$. (f) Woven nanotube arrays on a rectangular pattern, $50 \times 50 \mu \mathrm{m} ; Z$ scale is $80 \mathrm{~nm}$. The bottom inset shows 2D Fourier transform demonstrating perfect positional ordering of arrays. The top inset shows the "nest" shape of an individual array with nanotube bundles predominantly bent and aligned along the edges.

Inspection of the bent nanotubes at high magnification revealed that loops of different types formed by bending only a portion of the nanotube, typically less than half of its total length. A significant portion of the backward bent tube is in close contact with the straight, unbent portion. The radius of curvature of the coiled portion is 


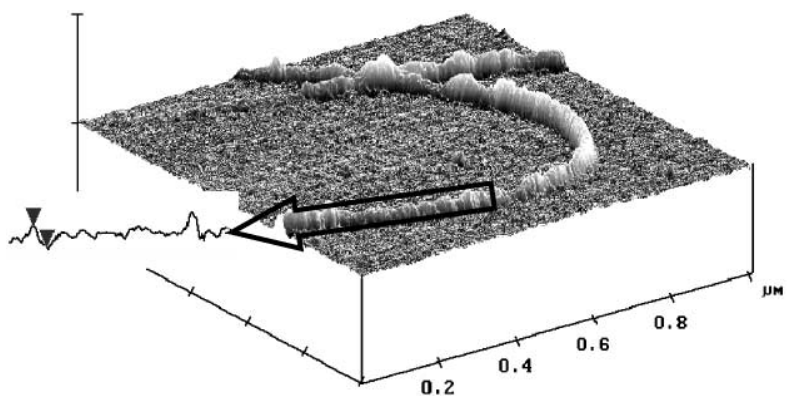

FIG. 3. High-resolution 3D images of looped nanotubes and cross-sectional profile along the bent nanotube (red marks indicate depth of $2.0 \mathrm{~nm}$ ). $Z$ scale is $20 \mathrm{~nm}$.

within $200-300 \mathrm{~nm}$. It is clear that bending and anchoring of the nanotubes under such confined conditions result in the development of significant local stresses, which lead to either the splitting of bundles or the sectioning of nanotubes (e.g., see 3D image of nanotube "arch" and surface profile in Fig. 3). In the last case, the flat surface sections of the nanotubes are separated by $0.3-0.6 \mathrm{~nm}$ grooves indicating a wrinkle formation. Overall, this results in one-dimensional roughness along the nanotube of $0.2-0.3 \mathrm{~nm}$, a characteristic of atomic scale corrugation of the nanotube walls caused by sharp wrinkles of the graphitic layers during bending as was already observed [18]. No single point buckling is found for the bent nanotubes indicating a uniform bending stress distributed over the entire nanotube length.

Considering the features of the phenomenon observed, we suggest the moving contact line to be responsible for the formation of woven or looped nanotubes (Fig. 4). In fact, the liquid drying under the unidirectional gravitational force inevitably leads to the directional flow within relatively thick layers followed by the formation of a gradient molecularly thick microfluidic layer, which is a subject of the contact line instability $[6,19,20]$. Such instabilities cause the formation of an array of periodically spaced microdroplets left behind the moving triple contact line [21].

To envision how this process could interact with carbon nanotubes, we should emphasize that the carbon nanotubes adsorbed from solution should be tethered to the hydrophilic surface [22]. The pinned nanotubes can be trapped by the moving contact line of drying microdroplets and initially align themselves along the droplet circumference in straight form (Fig. 4). At the next stage of drying, the decreasing of the overall length of the contact line along with increasing of its curvature force the trapped carbon nanotubes to follow the overall diminishing shape of the droplets by gradual bending (Fig. 4). The nanotube loops are fixed in this strained state by strong interactions with functionalized substrate and van der Waals interaction between the two portions of the nanotube in a close contact. The variation of the initial trapping scenario and size/location of a microdroplet causes a variety of looped shapes observed under different prepa-

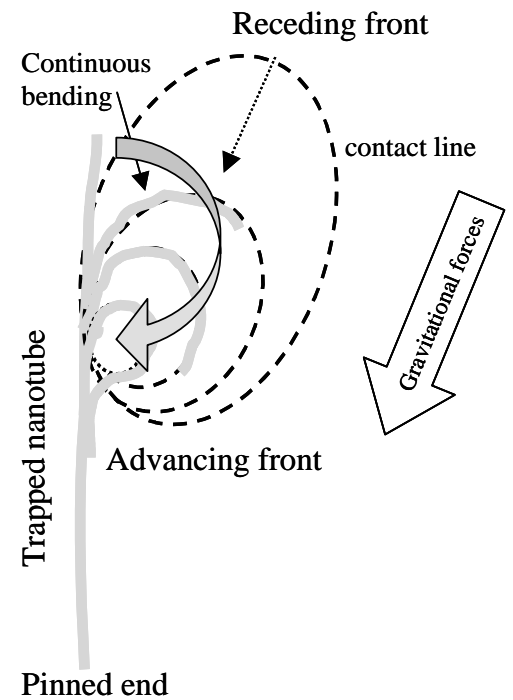

FIG. 4. Schematic of the suggested mechanism of pinned nanotube bending by a moving receding front of a drying droplet.

ration conditions. In addition, preorientation of carbon nanotubes along the hydrophilic stripes caused by hydrodynamic forces should play a significant role in the texture formation as will be discussed in a separate publication [23].

The mechanism of nanotube looping suggested here could be valid only if the surface tension acting along the shrinking contact line outweighs strain forces arising from nanotube bending. The evaluation of the surface tension, which arises from the shrinking contact line [24], leads to the conclusion that only elastic rods with a spring constant higher than $1-5 \mathrm{~N} / \mathrm{m}$ could sustain the pressure across the curved contact line. On the other hand, the evaluation of the bending properties required for nanotube folding can be conducted using the known value of the elastic modulus, $E \sim 1$ Tpa [25,26]. A spring constant $K$ for the nanotube portion participating in continuous bending can be determined within the solid rod approximation by using the equation $K=3 \pi R^{4} E / 4 L^{3}$, where $R$ is the bundle radius and $L$ is the length of the curved portion [27]. Estimation for $L=0.3-1 \mu \mathrm{m}$ and $R=1-2.5 \mathrm{~nm}$ gives values close to $10^{-2} \mathrm{~N} / \mathrm{m}$, which is well below the critical stiffness of $1-5 \mathrm{~N} / \mathrm{m}$ required to prevent tube bending. Thus, carbon nanotubes aligned along the fluid-air-solid interface can be easily bent by the shrinking contact line.

In fact, alignment, stretching, and bending of stiff rodlike molecules such as DNA aligned along the contact line were observed and used for the combing process and the evaluation of the nanomechanical elastic properties of the biological molecules $[28,29]$. On the other hand, the mechanism of trapping carbon nanotubes at the surface of microbubbles in ultrasonically treated solution and their bending during cavitation and bubble collapse was suggested as an explanation for the ring formation. 
Finally, we would like to emphasize the role of the surface patterning in organized coiling of the carbon nanotubes. Obviously, the initial formation of stripped microfluidic layers moving along the hydrophilic microscopic stripes is critical in stimulating the periodic instabilities of the contact line with spacing controlled by the dimensions of the hydrophilic and hydrophobic areas.

We believe that the combination of this newly observed phenomenon with proper hydrophilic-hydrophobic patterning may lead to directed assembly of uniformly oriented and looped nanotubes (carbon or other origin) in regular surface arrays. Controlled bending of nanotubes resulting in tunable electronic and optical properties can be thought as a means for the fabrication of large-scale nanosensor arrays.

Purification. - The single walled carbon nanotube (SWNT) raw material, produced by the arc discharge method, was purchased from MER (Arizona, U.S.A.). The purification procedure involved oxidation in nitric acid, centrifugation, and filtration [30]. SDS surfactant (Aldrich) was used to make stable colloidal suspension of SWNTs [31]. The suspension was filtered with polycarbonate membranes (pore size $0.4 \mu \mathrm{m}$ ).

Substrate patterning.-Microcontact printing $(\mu \mathrm{CP})$ was used for patterning of the silicon oxide surface [32]. The poly(dimethylsiloxane) (PDMS) stamp was cured and released from a patterned mold. Octadecyltrichlorosilane (OTS) solution was used as the ink for $\mu \mathrm{CP}$ with an aminopropyltriethoxysilane (APTS) layer formed on the unreacted regions. Procedures were conducted under Cleanroom-100 conditions according to usual procedure $[33,34]$.

Nanotube adsorption. - Casting was conducted by drop drying of nanotube solution on tilted substrates (Fig. 1). For the dip-coating process, patterned substrates were immersed in a nanotube solution for about $24 \mathrm{~h}$. After that, the substrates were withdrawn, dried vertically in air, and then rinsed.

Surface characterization. - AFM scanning was performed in the light tapping mode on a Dimension 3000 (Digital Instruments, Inc.) microscope under ambient conditions according to the usual experimental procedure adapted in our laboratory $[35,36]$.

This study is supported by NASA-CNDE Center at Iowa State University under Contract No. 102098, NSFCMS-0099868, and AFOSR Grant No. F496200210205.

*Author to whom correspondence should be addressed. Electronic address: vladimir@iastate.edu

[1] H. G. Craighead, Science 290, 1532 (2000).

[2] T.V. Sreekumar et al., Chem. Mater. 15, 175 (2003).
[3] K. Q. Jian, H.-S. Shim, A. Schwartzman, G. P. Crawford, and R. H. Hurt, Adv. Mater. 15, 164 (2003).

[4] H. Shimoda et al., Adv. Mater. 14, 899 (2002).

[5] M. Burghard, G. Duesberg, G. Philipp, J. Muster, and S. Roth, Adv. Mater. 10, 584 (1998).

[6] Y. Huang, X. Duan, Q. Wei, and C. M. Lieber, Science 291, 630 (2001).

[7] A. Cao et al., Appl. Phys. Lett. 81, 1297 (2002).

[8] J. Li, C. Papadopoulos, J. M. Xu, and M. Moskovits, Appl. Phys. Lett. 75, 367 (1999).

[9] A. Maiti, Nature Mater. 2, 440 (2003).

[10] A. Ural, Y. M. Li, and H. J. Dai, Appl. Phys. Lett. 81, 3464 (2002).

[11] S.V. Rotkin and Yu. Gogotsi, Mater. Res. Innov. 5, 191 (2002).

[12] G. M. Whitesides, J. P. Mathias, and C.T. Seto, Science 254, 1312 (1991).

[13] R. Martel, H. R. Shea, and P. Avouris, Nature (London) 398, 299 (1999).

[14] M. Sano, A. Kamino, J. Okamura, and S. Shinkai, Science 293, 1299 (2001).

[15] J. Liu et al., Chem. Phys. Lett. 303, 125 (1999).

[16] L. T. Cai, J. L. Bahr, Y. X. Yao, and J. M. Tour, Chem. Mater. 14, 4235 (2002).

[17] V. N. Bliznyuk, M. P. Everson, and V.V. Tsukruk, J. Tribol. 120, 489 (1998).

[18] Progress in Transmission Electron Microscopy 2. Applications in Materials Science, edited by X.-F. Zhang and Z. Zhang, Springer Series in Surface Sciences (Springer, New York, 2001), Vol. 39.

[19] S. M. Troian, X. L. Wu, and S. A. Safran, Phys. Rev. Lett. 62, 1496 (1989).

[20] J. A. Diez and L. Kondic, Phys. Rev. Lett. 86, 632 (2001).

[21] O. Kathaus, L. Gracjo, N. Maruyama, and M. Shimomura, Chaos 9, 308 (1999).

[22] B. Kim and W. M. Sigmund, Langmuir 19, 4848 (2003).

[23] H. Ko, S. Peleshanko, and V.V. Tsukruk, J. Phys. Chem. (to be published).

[24] A.W. Adamson, Physical Chemistry of Surfaces (Wiley, New York, 1990), 5th ed.

[25] E.W. Wong, P. E. Sheehan, and C. M. Lieber, Science 277, 1971 (1997).

[26] D. A. Walters et al., Appl. Phys. Lett. 74, 3803 (1999).

[27] F. P. Beer and E. R. Johnston, Mechanics of Materials (McGraw-Hill, New York, 1992).

[28] A. Bensimon et al., Science 265, 2096 (1994).

[29] D. Bensimon, A. J. Simon, V. Croquette, and A. Bensimon, Phys. Rev. Lett. 74, 4754 (1995).

[30] J. Liu et al., Science 280, 1253 (1998).

[31] J.-M. Bonard, Adv. Mater. 9, 827 (1997).

[32] N. L. Jeon, K. Finnie, K. Branshaw, and R. G. Nuzzo, Langmuir 13, 3382 (1997).

[33] V.V. Tsukruk and V. N. Bliznyuk, Langmuir 14, 446 (1998).

[34] V.V. Tsukruk, Adv. Mater. 13, 95 (2001).

[35] V.V. Tsukruk and D. H. Reneker, Polymer 36, 1791 (1995).

[36] V.V. Tsukruk, Rubber Chem. Technol. 70, 430 (1997). 